\title{
Event-Driven Control for NCSs with Logarithmic Quantization and Packet Losses
}

\author{
Jingjing Yan \\ College of Electrical Engineering, Henan University of Technology, Zhengzhou 450052, China \\ Correspondence should be addressed to Jingjing Yan; yanjingjing2009@haut.edu.cn
}

Received 12 April 2017; Accepted 9 August 2017; Published 17 September 2017

Academic Editor: Petko Petkov

Copyright (C) 2017 Jingjing Yan. This is an open access article distributed under the Creative Commons Attribution License, which permits unrestricted use, distribution, and reproduction in any medium, provided the original work is properly cited.

\begin{abstract}
The stabilization problem of the networked control systems (NCSs) affected by data quantization, packet losses, and event-driven communication is studied in this paper. By proposing two event-driven schemes and the extended forms of them relying on quantized states, zoom strategy is adopted here to study the system stability with time-varying logarithmic quantization and independent identically distributed (IID) packet losses process. On the basis of that, some sufficient conditions ensuring the mean square stability of the system are obtained here. Although zoom strategy has been utilized by many literatures to study the quantized stabilization problem of NCSs, it has not been adopted to analyze the stability of NCSs with data quantization, IID packet losses, and event-driven communication. Furthermore, the existing literatures relating to zoom strategy employ the quantizer with quantization regions holding arbitrary shapes, but here we use the logarithmic quantizer which holds better performance near the origin. In addition, the detailed comparisons of the system performance under different event-driven schemes are given here, which can guide the strategy selection according to the different design goals. The above three points are the main innovations of this paper. At last, the effectiveness of the proposed methods is illustrated by a benchmark example.
\end{abstract}

\section{Introduction}

The introduction of the network in the control system brings us a lot of convenience, such as increased flexibility and maintainability and reduced wiring cost. However, network inserted in the control system also brings some new challenges for studying the stability and stabilization problems of the closed-loop system. Under the network-induced imperfections including data quantization, transmission delay, packet losses, and communication constraints, the sampled signals can not be transmitted timely and accurately through the network, which results in the fact that the system performance may be degraded, or even instability. How to design suitable controller based on noncomplete information to stabilize the NCSs has gotten a lot of attention in recent years.

As two important issues in NCSs, quantization and packet losses have been researched by many literatures including [1-11]. Ji et al. study the mean square quadratic stabilization problem for NCSs with arbitrary packet losses and Markovian jump packet losses, respectively, in [1] and [2]. An optimal quantizer/controller design method is represented in [3]. For the systems with multiple networks, literature in [4] proposes a less conservative sufficient stabilizing bit rate condition. The problem of optimal tracking of multi-input multioutput systems with quantization and packet-dropouts is studied in [5]. Literature in [6] discusses the $H_{\infty}$ quantized control problem for the neural network affected by distributed time delay and packet losses. For the linear systems and fuzzy systems affected by delays, quantization, and packet dropout, observer-based output feedback controllers are designed in [7] and [8], respectively. Moreover, the design criteria for the static output feedback controller, optimal dynamic quantizer, and $H_{\infty}$ controller are researched in [9], [10], and [11], respectively.

Some special methods have been adopted to deal with quantization and packet losses issues, such as [12-19]. A sliding mode controller is designed in literature [12] to stabilize the NCSs. A switched dynamic controller is proposed in [13] by converting the system into a time-delay switched system. By adopting model predictive control method, literatures in [14] and [15] discuss the issues of quantization and packet losses for nonlinear systems and constrained feedback control 
systems, respectively. Literature in [16] also discusses the effect of time-varying delay on system performance by quantized predictive control method. By utilizing zoom strategy, we discuss the quantized control for linear systems and fuzzy systems with packet losses in [17] and [18], respectively, and consider the unknown disturbances simultaneously in [19].

Event-driven scheme is a useful method to reduce the communication resources in contrast with the periodic protocol, which means that just important signals are sent through the network; that is, if the signals being sampled have not altered sufficiently, there is no superiority to transfer them. Some papers have discussed the quantized control for the systems with event-driven scheme, such as [2022]. By adopting an event-driven zero order holder to deal with the effects of stochastic packet losses, the stochastic stability of the system under quantized control is discussed in [20]. Under the event-driven communication, literature in [21] studies the synchronization for networked passive systems with data quantization. For the hybrid NCSs, [22] derives a sufficient condition of the stochastic stability by proposing a distributed event-triggered scheme and multiple quantization method.

The purpose of this paper is studying the issues of quantization and packet losses for NCSs under event-driven communication. In fact, the problem studied here has been researched in $[23,24]$. By restraining the upper bound of the number of successive packet losses and adopting the logarithmic quantization strategy proposed in $[25,26]$, the sufficient conditions ensuring the asymptotical stability of the systems are shown in [23], in which an event-driven scheme is designed by the errors between the current sampling states and the last transmitted states. The differences of this paper with [23] include three aspects. (i) In this paper, the packet losses are modeled as an IID process, under which the mean square stability rather than the asymptotical stability can be guaranteed. (ii) Although logarithmic quantizer is also used in our paper, the parameters of which are time-varying based on zoom strategy. However, the parameters in [23] are all fixed. (iii) The event-driven scheme proposed here is according to the error between the current (quantized) sampling states and the last transmitted quantized states, which is more reasonable than [23]. Moreover, the stabilization problem discussed here is also studied in [24], where the quantized controller was given for NCSs with eventdriven communication by modeling the Markov packet losses process. The different points between this paper and [24] include the following: (i) the models of packet losses are different from each other. (ii) Zoom strategy is used in both our paper and [24]. However, quantizer used in [24] is proposed in [27], but the one we adopted is a logarithmic quantizer proposed in $[25,26]$. (iii) Only one event-driven scheme is adopted in [24]. But in this paper, we propose two event-driven schemes and compare them with each other. Furthermore, we also extend the event-driven schemes to the ones relating to an additional variable $m$, the effect of which on the system performance is also studied.

Summarized above, our contributions with respect to earlier literatures are three aspects.
(1) Depending on the quantized states, two event-driven schemes and the extended forms of them are proposed in this paper, which are more applicable due to the quantized data being indeed transmitted through the network under the limited bandwidth.

(2) The detailed comparison of the event-driven schemes is given here, based on which users can choose different strategies according to their actual needs including minimizing transmission times, increasing convergence rate, or extending the applicable scope of the algorithm.

(3) Although there are many literatures using zoom strategy to deal with data quantization, the quantizer adopted there is the one proposed in [27]. Considering the advantages of the logarithmic quantizer, such as which holds better performance near the origin, we adopt zoom strategy to handle the stabilization problem for NCSs with logarithmic quantization, packet losses, and event-driven communication. It is worth mentioning that the logarithmic quantization combined with zoom strategy has already been studied in our paper [28], but in which the packet losses and event-driven scheme are all uncovered.

The organization of our paper is arranged as follows. The problem formulation including the detailed system, network, and closed-loop system formulations is described in Section 2. Mean square stabilities of the closed-loop system under two event-driven schemes are studied in Sections 3 and 4 . Some analysis and comparison results are illustrated in Section 5. Simulation and conclusion are given, respectively, in Sections 6 and 7.

Notation. $n$-dimensional Euclidean space is denoted as $\mathbb{R}^{n}$. $\mathbb{R}^{+}$and $\mathbb{N}$ represent the positive real numbers set and positive integers set, respectively. The Euclidean norm in $\mathbb{R}^{n}$ and corresponding induced matrix norm in $\mathbb{R}^{n \times n}$ are both illustrated by $\|\cdot\|$. For the positive-definite matrix $P, \lambda_{\max }(P)$ and $\lambda_{\min }(P)$ are, respectively, used to denote the maximum and minimum eigenvalues of it. The transposed matrix $A \in$ $\mathbb{R}^{n \times m}$ is indicated by $A^{\top} \in \mathbb{R}^{m \times n}$. The least integer not less than $y$ is signified by the signal $\lceil y\rceil$. Zero vector with dimension $n \times 1$ is denoted as $\mathbf{0}_{n \times 1}$.

\section{Problem Formulation}

2.1. System Formulation. We considers the following continuous-time system:

$$
\dot{x}(t)=A_{c} x(t)+B_{c} u(t)
$$

where $x \in \mathbb{R}^{n}$ is the system state, $u \in \mathbb{R}^{n \times m}$ denotes the control input, and $A_{c}$ and $B_{c}$ are constant matrices with appropriate dimensions. Given sampling period $T$, system (1) can be rewritten as discrete-time one:

$$
x(k+1)=A x(k)+B u(k)
$$

with $A=e^{A_{c} T}$ and $B=\int_{0}^{T} e^{A_{c} t} B_{c} d t$, where $t$ represents continuous-time instant and $k$ is the time step satisfying $t_{k}=$ $k T$. 


\subsection{Network Formulation}

2.2.1. Packet Losses. The packet losses process is modeled as an IID process, in which $\xi(k)=1$ denotes the successful transmission of data packets at time instant $k$, and $\xi(k)=$ 0 represents the occurrence of packet losses. Moreover, we assume that the random variable $\xi(k)$ satisfies $\operatorname{Pr}(\xi(k)=0)=$ $\beta$ and $\operatorname{Pr}(\xi(k)=1)=1-\beta$.

2.2.2. Data Quantization. For the $i$ th component of the state $x(k), i \in\{1,2, \ldots, n\}$, the time-varying logarithmic quantizer adopted in this paper is represented as

$$
q\left(x_{i}(k)\right)= \begin{cases}w_{j} & \text { if } x_{i}(k) \in\left[\frac{1}{1+\delta} w_{j},+\infty\right) \\ w_{j+m} & \text { if } x_{i}(k) \in\left[\frac{1}{1+\delta} w_{j+m}, \frac{1}{1-\delta} w_{j+m}\right), m \in\{1,2, \ldots, N-1\} \\ 0 & \text { if } x_{i}(k) \in\left[0, \frac{1}{1+\delta} w_{j+N-1}\right) \\ -q\left(-x_{i}(k)\right) & \text { if } x_{i}(k) \in(-\infty, 0]\end{cases}
$$

with $\delta \in(0,1), w_{j+1}=\rho w_{j}, w_{0}>0, \rho=(1-\delta) /(1+\delta) \epsilon$ $(0,1)$ for any $k \in\left[k_{j}, k_{j+1}\right), j \in \mathbb{N} \cup\{0\}$. It is obvious that $\delta, \rho, w_{0}$, and $k_{j}$ are the critical parameters of the logarithmic quantizer, which are all assumed to be undetermined. On the basis of $q\left(x_{i}(k)\right), i \in\{1,2, \ldots, n\}$, the quantization value of the vector $x(k)$ is defined as

$$
Q(x(k))=\left[q\left(x_{1}(k)\right), \ldots, q\left(x_{n}(k)\right)\right]^{\top} .
$$

Remark 1. Existing articles involving zoom strategy mostly use the quantizer defined in literature in [27]. Using logarithmic quantizer to deal with quantization and packet losses based on zoom strategy is one of the innovations of this paper.

2.2.3. Event-Driven Scheme. To reduce the network burden, we set the sampling data to be transmitted just at some specific times. Denote the transmission times as $i_{j}$ with $j, i_{j} \in$ $\mathbb{N} \cup\{0\}$ satisfying $i_{0}=0, i_{j}<i_{j+1}$. Two event-driven schemes are designed in this paper, in which $i_{j+1}$ is selected, respectively, as

$$
\begin{array}{r}
i_{j+1} \\
=\min \left\{k>i_{j} \mid\left\|Q\left(x\left(i_{j}\right)\right)-Q(x(k))\right\|>\gamma(k)\right\}, \\
k \in \mathbb{N} \cup\{0\}, \\
i_{j+1}=\min \left\{k>i_{j} \mid\left\|Q\left(x\left(i_{j}\right)\right)-x(k)\right\|>\gamma(k)\right\}, \\
k \in \mathbb{N} \cup\{0\}
\end{array}
$$

in which $\gamma(k)$ is a piecewise constant function denoted by

$$
\begin{aligned}
\gamma(k)=\sqrt{n} & \frac{\delta}{1-\delta} w_{l}, \\
& \forall k \in\left[k_{0}+l \theta, k_{0}+(l+1) \theta\right), l \in \mathbb{N} \cup\{0\},
\end{aligned}
$$

where $k_{0}$ and $\theta$ are defined below.
2.3. Closed-Loop System. Under the above network constraints and event-driven schemes, the controller is defined as

$$
\begin{aligned}
& u(k)=\xi(k) K Q\left(x\left(i_{j}\right)\right) \\
& \forall k \in\left[i_{j}, i_{j+1}\right), j, i_{j} \in \mathbb{N} \cup\{0\} .
\end{aligned}
$$

The following closed-loop system can be obtained by combining (2) and (7):

$$
x(k+1)=A x(k)+\xi(k) B K Q\left(x\left(i_{j}\right)\right) .
$$

The purpose of this paper is proposing suitable design methods for logarithmic quantizer such that the system (8) with the above-mentioned network-induced effects and event-driven schemes (5a) and (5b) to achieve the mean square stability.

\section{Stability Analysis for the System (8) under Event-Driven Scheme (5a)}

The aim of this section is to design suitable quantizer parameters including $\delta, \rho, w_{0}$, and $k_{j}$, under which the mean square stability of the system (8) under event-driven scheme (5a) is guaranteed. To obtain this purpose, we rewrite (8) as

$$
\begin{aligned}
x(k+1)= & (A+B K) x(k)-(1-\xi(k)) B K x(k) \\
& +\xi(k) B K e(k)+\xi(k) B K g(k)
\end{aligned}
$$

with $e(k)=Q(x(k))-x(k)$ and $g(k)=Q\left(x\left(i_{j}\right)\right)-Q(x(k))$.

Theorem 2. Assume that the packet loss rate $\beta$ is given, if there are positive-definite matrix $\Xi_{1}$ and matrix $\Psi_{1}$ satisfying

$$
\left[\begin{array}{ccc}
-\Xi_{1} & \left(A \Xi_{1}+B \Psi_{1}\right)^{\top} & \left(B \Psi_{1}\right)^{\top} \\
* & -\frac{1}{4} \Xi_{1} & 0 \\
* & * & -\frac{1}{2 \beta} \Xi_{1}
\end{array}\right]<0
$$


then the feedback matrix $K$ defined by $K=\Psi_{1} P$ with $P=\Xi_{1}^{-1}$ can ensure that the system (8) is mean square stability under event-driven scheme (5a) if quantizer parameters $\delta, \rho$, and $k_{j}=k_{0}+j \theta, j \in \mathbb{N}$ are selected satisfying $\delta>\rho^{N}$ and

$$
\begin{aligned}
& \rho=\sqrt{\frac{\lambda_{\max }(P)}{\lambda_{\text {min }}(P)}} \sqrt{2 n(1+\pi)} \Phi_{1} \delta<1, \\
& \theta=\lceil\tilde{\theta}\rceil=\left\lceil\frac{\lambda_{\min }(P)-\lambda_{\max }(P) 2 n(1+\pi) \Phi_{1}^{2} \delta^{2}}{\eta_{1} 2 n \pi \Phi_{1}^{2} \delta^{2}}\right\rceil
\end{aligned}
$$

for any given $\pi \in \mathbb{R}^{+}$, where $\Phi_{1}$ and $\eta_{1}$ are, respectively, given as $\Phi_{1}=\left(\left(3(1-\beta) / \eta_{1}\right)\left\|K^{\top} B^{\top} P B K\right\|\right)^{1 / 2}$ and $\eta_{1}=\lambda_{\min }\left(\Psi_{1}\right)$ with $\Psi_{1}=P-4(A+B K)^{\top} P(A+B K)-2 \beta K^{\top} B^{\top} P B K$. Moreover, the variables $w_{0}$ and $k_{0}$ are determined by (15).

Proof. Let $V(x(k))=E\left\{x^{\top}(k) P x(k)\right\}$. Taking the fact $\xi(k)(1-$ $\xi(k))=0$ into consideration, we get

$$
\begin{aligned}
x^{\top}(k & +1) P x(k+1) \\
\leq & 4 x^{\top}(k)(A+B K)^{\top} P(A+B K) x(k) \\
& +2(1-\xi(k))^{2} x^{\top}(k) K^{\top} B^{\top} P B K x(k) \\
& +3 \xi^{2}(k) e^{\top}(k) K^{\top} B^{\top} P B K e(k) \\
& +3 \xi^{2}(k) g^{\top}(k) K^{\top} B^{\top} P B K g(k)
\end{aligned}
$$

by the inequalities $E^{\top} P F+F^{\top} P E \leq E^{\top} P E+F^{\top} P F$ and $-E^{\top} P F-F^{\top} P E \leq E^{\top} P E+F^{\top} P F$ for any matrices $E, F$, and positive-definite matrix $P$. Combine with (12) and the facts $E\left\{(1-\xi(k))^{2}\right\}=\beta$ and $E\left\{\xi(k)^{2}\right\}=1-\beta$, which directly derives that

$$
\begin{aligned}
\Delta V & (x(k))=E\left\{x^{\top}(k+1) P x(k+1)\right\}-x^{\top}(k) P x(k) \\
& \leq x^{\top}(k) \\
& \cdot\left(4(A+B K)^{\top} P(A+B K)+2 \beta K^{\top} B^{\top} P B K-P\right) \\
& \cdot x(k)+3(1-\beta) e^{\top}(k) K^{\top} B^{\top} P B K e(k) \\
& +3(1-\beta) g^{\top}(k) K^{\top} B^{\top} P B K g(k) .
\end{aligned}
$$

If the linear matrix inequality (10) holds, then it must give a positive-definite matrix $\Psi_{1}$ satisfying $4(A+B K)^{\top} P(A+B K)+$ $2 \beta K^{\top} B^{\top} P B K-P=-\Psi_{1}<0$. Let $\eta_{1}=\lambda_{\text {min }}\left(\Psi_{1}\right)$; then the inequality (13) gives us

$$
\begin{aligned}
\Delta V(x(k)) & \\
\leq & -\eta_{1}\|x(k)\|^{2} \\
& +3(1-\beta)\left\|K^{\top} B^{\top} P B K\right\|\left(\|e(k)\|^{2}+\|g(k)\|^{2}\right) .
\end{aligned}
$$

Zooming-Out Stage. In this stage, it set the system as uncontrolled form. The aim is designing a suitable quantizer parameter $w_{0}$ such that it gives a time instant $k_{0}$, at which the system state is unsaturated, that is, $\left\|x\left(k_{0}\right)\right\| \leq w_{0} /(1-\delta)$. To achieve this purpose, we let $\widetilde{w}(k+1)=\left(1 / \rho^{\varphi}\right) \widetilde{w}(k), \forall k \in$ $\mathbb{N} \cup\{0\}$, where $\widetilde{w}(0)$ is an arbitrary given positive real number and the selection of $\varphi$ satisfies $\rho^{\varphi}<\|A\|^{-1}<1$. Hence there must exist a time instant $k_{0}$ satisfying

$$
\left\|x\left(k_{0}\right)\right\| \leq \sqrt{\frac{\lambda_{\min }(P)}{\lambda_{\max }(P)}} \frac{\widetilde{w}\left(k_{0}\right)}{1-\delta}:=\sqrt{\frac{\lambda_{\min }(P)}{\lambda_{\max }(P)}} \frac{w_{0}}{1-\delta},
$$

indicating that

$$
\begin{aligned}
& x\left(k_{0}\right) \in R_{0} \\
& \quad:=\left\{x(k): x^{\top}(k) P x(k) \leq\left(\frac{w_{0}}{1-\delta}\right)^{2} \lambda_{\min }(P)\right\}
\end{aligned}
$$

and thus $\left\|x\left(k_{0}\right)\right\| \leq w_{0} /(1-\delta)$ hold, which accomplishes the goals of this stage.

Zooming-In Stage. At time instant $k_{0}$, zoom strategy is transferred to zooming-in stage, and the mode of the system is changed from open-loop to closed-loop. The aim of this stage is to design appropriate quantizer parameters $\rho, \delta$ and time instants $k_{j}, j \in \mathbb{N}$ such that $E\{\|x(k)\|\} \leq w_{j} /(1-\delta), \forall k \in$ $\left[k_{j}, k_{j+1}\right)$. In order to obtain this aim, we first prove that $R_{0}$ is an invariant set. In fact, if $x(k) \in R_{0}$, then $\|x(k)\| \leq w_{0} /(1-\delta)$, and thus $\left|x_{i}(k)\right| \leq w_{0} /(1-\delta)$ and $\left|q\left(x_{i}(k)\right)-x_{i}(k)\right| \leq(\delta /(1-$ $\delta)) w_{0}$ hold for any $i \in\{1,2, \ldots, n\}$ by using $\delta>\rho^{N}$. Hence we get

$$
\|e(k)\| \leq \sqrt{n} \frac{\delta}{1-\delta} w_{0}
$$

Moreover, event-driven scheme (5a) tells us

$$
\|g(k)\| \leq \gamma(k) \leq \sqrt{n} \frac{\delta}{1-\delta} w_{0} .
$$

Combined with (14), (17), and (18), we see

$$
\begin{aligned}
\Delta V(x(k)) & \\
\leq & -\eta_{1}\|x(k)\|^{2} \\
& +3(1-\beta)\left\|K^{\top} B^{\top} P B K\right\| 2 n\left(\frac{\delta}{1-\delta} w_{0}\right)^{2} .
\end{aligned}
$$

Thus the set $\mathbf{B}$ defined by

$$
\mathbf{B}=\left\{x(k):\|x(k)\| \leq \Phi_{1} \sqrt{2 n} \frac{\delta}{1-\delta} w_{0}\right\}
$$

with $\Phi_{1}=\left(\left(3(1-\beta) / \eta_{1}\right)\left\|K^{\top} B^{\top} P B K\right\|\right)^{1 / 2}$ is obviously an invariant set. If we define

$$
\begin{aligned}
\widetilde{B} & =\left\{x(k): x^{\top}(k) P x(k)\right. \\
& \left.\leq \lambda_{\max }(P) \Phi_{1}^{2} 2 n\left(\frac{\delta}{1-\delta} w_{0}\right)^{2}\right\}
\end{aligned}
$$


and select $\delta$ satisfying $\sqrt{\lambda_{\max }(P) / \lambda_{\min }(P)} \Phi_{1} \sqrt{2 n} \delta \sqrt{1+\pi}<$ 1 , where $\pi \in \mathbb{R}^{+}$, then it must be $R_{0} \supset \widetilde{B} \supset \mathbf{B}$, and hence $R_{0}$ is an invariant set.

By defining

$$
\widetilde{\theta}=\frac{\lambda_{\min }(P)-\lambda_{\max }(P) 2 n(1+\pi) \Phi_{1}^{2} \delta^{2}}{\eta_{1} 2 n \pi \Phi_{1}^{2} \delta^{2}}
$$

and $\theta=\lceil\widetilde{\theta}\rceil$, we will next prove

$$
E\left\{\left\|x\left(k_{1}\right)\right\|\right\} \leq \frac{1}{1-\delta} w_{1}
$$

with $k_{1}=k_{0}+\theta$ if $\rho$ is selected as $\rho=$ $\sqrt{\lambda_{\max }(P) / \lambda_{\min }(P)} \Phi_{1} \sqrt{2 n} \delta \sqrt{1+\pi}$. In order to achieve this purpose, we will first prove

$$
\begin{aligned}
& E\left\{x^{\top}\left(k_{1}\right) P x\left(k_{1}\right)\right\} \\
& \quad \leq \lambda_{\max }(P) 2 n(1+\pi)\left(\Phi_{1} \frac{\delta}{1-\delta} w_{0}\right)^{2} .
\end{aligned}
$$

If we assume that (24) is unsatisfied, this means

$$
\begin{aligned}
& E\left\{x^{\top}\left(k_{1}\right) P x\left(k_{1}\right)\right\} \\
& \quad>\lambda_{\max }(P) 2 n(1+\pi)\left(\Phi_{1} \frac{\delta}{1-\delta} w_{0}\right)^{2} .
\end{aligned}
$$

Defining the set $\widetilde{R}_{1}$ as

$$
\begin{aligned}
\widetilde{R}_{1} & =\left\{x(k): E\left\{x^{\top}(k) P x(k)\right\}\right. \\
& \left.\leq \lambda_{\max }(P) 2 n(1+\pi)\left(\Phi_{1} \frac{\delta}{1-\delta} w_{0}\right)^{2}\right\},
\end{aligned}
$$

then it must be $\widetilde{R}_{1} \supset \mathbf{B}$. This gives the fact that $\widetilde{R}_{1}$ is an invariant set, which combined with (25) tells us

$$
\begin{aligned}
& E\left\{x^{\top}(k) P x(k)\right\} \\
& \quad>\lambda_{\max }(P) 2 n(1+\pi)\left(\Phi_{1} \frac{\delta}{1-\delta} w_{0}\right)^{2},
\end{aligned}
$$

and thus $E\left\{\|x(k)\|^{2}\right\}>2 n(1+\pi)\left(\Phi_{1}(\delta /(1-\delta)) w_{0}\right)^{2}$ for any $k \in\left[k_{0}, k_{1}\right)$. Similar to the analysis of (14)-(19), we know that

$$
\begin{aligned}
\Delta V & \left(x\left(k_{0}+\theta-\varsigma\right)\right) \\
& =E\left\{x^{\top}\left(k_{0}+\theta-\varsigma+1\right) P x\left(k_{0}+\theta-\varsigma+1\right)\right. \\
& \left.-x^{\top}\left(k_{0}+\theta-\varsigma\right) P x\left(k_{0}+\theta-\varsigma\right)\right\} \\
& \leq-\eta_{1} E\left\{\left\|x\left(k_{0}+\theta-\varsigma\right)\right\|^{2}\right\} \\
& +\eta_{1} 2 n \Phi_{1}^{2}\left(\frac{\delta}{1-\delta} w_{0}\right)^{2}<-\eta_{1} 2 n \Phi_{1}^{2}\left(\frac{\delta}{1-\delta} w_{0}\right)^{2}(1 \\
& +\pi)+\eta_{1} 2 n \Phi_{1}^{2}\left(\frac{\delta}{1-\delta} w_{0}\right)^{2} \\
& =-\eta_{1} 2 n \Phi_{1}^{2}\left(\frac{\delta}{1-\delta} w_{0}\right)^{2} \pi
\end{aligned}
$$

for any $\varsigma \in\{1,2, \ldots, \theta\}$. Hence we get

$$
\begin{aligned}
& E\left\{x^{\top}\left(k_{0}+\theta\right) P x\left(k_{0}+\theta\right)\right\}-x^{\top}\left(k_{0}\right) P x\left(k_{0}\right) \\
&<-\eta_{1} 2 n \Phi_{1}^{2}\left(\frac{\delta}{1-\delta} w_{0}\right)^{2} \pi \theta \\
&<-\eta_{1} 2 n \Phi_{1}^{2}\left(\frac{\delta}{1-\delta} w_{0}\right)^{2} \pi \tilde{\theta} \\
&<\lambda_{\max }(P) 2 n(1+\pi) \Phi_{1}^{2}\left(\frac{\delta}{1-\delta} w_{0}\right)^{2} \\
& \quad-\lambda_{\min }(P)\left(\frac{1}{1-\delta} w_{0}\right)^{2} .
\end{aligned}
$$

But (16) and (25) give us

$$
\begin{gathered}
E\left\{x^{\top}\left(k_{0}+\theta\right) P x\left(k_{0}+\theta\right)\right\}-x^{\top}\left(k_{0}\right) P x\left(k_{0}\right) \\
>\lambda_{\max }(P) 2 n(1+\pi) \Phi_{1}^{2}\left(\frac{\delta}{1-\delta} w_{0}\right)^{2} \\
\quad-\lambda_{\min }(P)\left(\frac{1}{1-\delta} w_{0}\right)^{2},
\end{gathered}
$$

which contradicts formula (29). So the inequality (24) must be established, which means

$$
E\left\{x^{\top}\left(k_{1}\right) P x\left(k_{1}\right)\right\} \leq \lambda_{\min }(P)\left(\frac{w_{1}}{1-\delta}\right)^{2},
$$

indicating that (23) holds. Moreover, we define $R_{1}$ as

$$
\begin{aligned}
& R_{1} \\
& =\left\{x(k): E\left\{x^{\top}(k) P x(k)\right\} \leq \lambda_{\text {min }}(P)\left(\frac{w_{1}}{1-\delta}\right)^{2}\right\} .
\end{aligned}
$$

Similar to the analysis of $R_{0}$, it is easy to see that $R_{1}$ is an invariant set and $x(k) \in R_{1}, \forall k \in\left[k_{1}, k_{2}\right)$. Similarly, for any $j \in \mathbb{N}$, by defining

$$
\begin{aligned}
& R_{j} \\
& \quad=\left\{x(k): E\left\{x^{\top}(k) P x(k)\right\} \leq \lambda_{\text {min }}(P)\left(\frac{w_{j}}{1-\delta}\right)^{2}\right\} ;
\end{aligned}
$$

we get that $x(k) \in R_{j}, \forall k \in\left[k_{j}, k_{j+1}\right)$ holds, and thus $E\left\{\|x(k)\|^{2}\right\} \leq\left(w_{j} /(1-\delta)\right)^{2}, \forall k \in\left[k_{j}, k_{j+1}\right)$. Taking the limit on both sides of above inequality, it gives $\lim _{k \rightarrow \infty} E\left\{\|x(k)\|^{2}\right\} \leq \lim _{j \rightarrow \infty}\left(w_{j} /(1-\delta)\right)^{2}=0$. This completes the proof.

\section{Stability Analysis for System (8) under Event-Driven Scheme (5b)}

In order to design quantizer parameters $\delta, \rho, w_{0}$, and $k_{j}$ such that the system (8) under event-driven scheme (5b) is mean square stability, we first rewrite the system (8) as

$$
\begin{aligned}
x(k+1)= & (A+B K) x(k)+\xi(k) B K f(k) \\
& -(1-\xi(k)) B K x(k)
\end{aligned}
$$


with $f(k)=Q\left(x\left(i_{j}\right)\right)-x(k)$, by which the following theorem can be derived.

Theorem 3. We assume that the packet loss rate $\beta$ is fixed, if it gives positive-definite matrix $\Xi_{2}$ and matrix $\Psi_{2}$ such that

$$
\left[\begin{array}{ccc}
-\Xi_{2} & \left(A \Xi_{2}+B \Psi_{2}\right)^{\top} & \left(B \Psi_{2}\right)^{\top} \\
* & -\frac{1}{3} \Xi_{2} & 0 \\
* & * & -\frac{1}{2 \beta} \Xi_{2}
\end{array}\right]<0 ;
$$

then the mean square stability of the system (8) with eventdriven scheme (5b) under the feedback matrix $K$ defined by $K=\Psi_{2} P$ with $P=\Xi_{2}^{-1}$ can be ensured if quantizer parameters $\delta, \rho$, and $k_{j}=k_{0}+j \theta, j \in \mathbb{N}$, satisfy $\delta>\rho^{N}$ and

$$
\begin{aligned}
& \rho=\sqrt{\frac{\lambda_{\max }(P)}{\lambda_{\min }(P)}} \sqrt{n(1+\pi)} \Phi_{2} \delta<1, \\
& \theta=\lceil\tilde{\theta}\rceil=\left\lceil\frac{\lambda_{\min }(P)-\lambda_{\max }(P) n(1+\pi) \Phi_{2}^{2} \delta^{2}}{\eta_{2} n \pi \Phi_{2}^{2} \delta^{2}}\right\rceil
\end{aligned}
$$

for any given $\pi \in \mathbb{R}^{+}$, where $\Phi_{2}$ and $\eta_{2}$ are, respectively, given as $\Phi_{2}=\left(\left(2(1-\beta) / \eta_{2}\right)\left\|K^{\top} B^{\top} P B K\right\|\right)^{1 / 2}$ and $\eta_{2}=\lambda_{\text {min }}\left(\Psi_{2}\right)$ with $\Psi_{2}=P-3(A+B K)^{\top} P(A+B K)-2 \beta K^{\top} B^{\top} P B K$. Moreover, the variables $w_{0}$ and $k_{0}$ are also described by (15).

Proof. Set $V(x(k))=E\left\{x^{\top}(k) P x(k)\right\}$; direct calculations give

$$
\begin{aligned}
& \Delta V(x(k))=E\left\{x^{\top}(k+1) P x(k+1)\right\}-x^{\top}(k) P x(k) \\
& \quad \leq x^{\top}(k) \\
& \cdot\left(3(A+B K)^{\top} P(A+B K)+2 \beta K^{\top} B^{\top} P B K-P\right) \\
& \cdot x(k)+2(1-\beta) f^{\top}(k) K^{\top} B^{\top} P B K f(k) .
\end{aligned}
$$

If (35) is satisfied, there must exist a positive-definite matrix $\Psi_{2}$ such that

$$
\begin{aligned}
& 3(A+B K)^{\top} P(A+B K)+2 \beta K^{\top} B^{\top} P B K-P=-\Psi_{2} \\
& \quad<0 .
\end{aligned}
$$

Let $\eta_{2}=\lambda_{\text {min }}\left(\Psi_{2}\right)$; from (37) and (38) we can easily get

$$
\begin{aligned}
\Delta V(x(k)) \leq & -\eta_{2}\|x(k)\|^{2} \\
& +2(1-\beta)\left\|K^{\top} B^{\top} P B K\right\|\|f(k)\|^{2} .
\end{aligned}
$$

As can be seen from the above formula, there is no need to discuss the impact of quantization errors on system stability in the following analysis. However, since the quantizer has a saturation boundary, the distance between $x(k)$ and $Q\left(x\left(i_{j}\right)\right)$ may always be greater than $\gamma(k)$. In order to ensure that events under the event-driven scheme (5b) are not triggered frequently, it is necessary to discuss and restrain the range of values for $x(k)$ in the analysis process. Based on this, the selection method of $k_{0}$ in the zooming-out stage of Theorem 2 is adopted here to ensure the boundedness of $x\left(k_{0}\right)$; that is,

$$
\begin{aligned}
& x\left(k_{0}\right) \in R_{0} \\
& \quad:=\left\{x(k): x^{\top}(k) P x(k) \leq\left(\frac{w_{0}}{1-\delta}\right) \lambda_{\min }(P)\right\} .
\end{aligned}
$$

Combining with (39) and (40) gives us

$$
\begin{aligned}
\Delta V(x(k)) & \\
\leq & -\eta_{2}\|x(k)\|^{2} \\
& +2(1-\beta)\left\|K^{\top} B^{\top} P B K\right\| n\left(\frac{\delta}{1-\delta} w_{0}\right)^{2} .
\end{aligned}
$$

With the analytical ideas of Theorem 2, we obtain that if variables $\Phi_{2}, \rho, \theta$, and $k_{j}, j \in \mathbb{N}$ are defined as

$$
\begin{aligned}
\Phi_{2} & =\left(\frac{2(1-\beta)}{\eta_{2}}\left\|K^{\top} B^{\top} P B K\right\|\right)^{1 / 2}, \\
\rho & =\sqrt{\frac{\lambda_{\max }(P)}{\lambda_{\min }(P)}} \sqrt{n(1+\pi)} \Phi_{2} \delta<1, \\
\theta & =\left\lceil\frac{\lambda_{\text {min }}(P)-\lambda_{\max }(P) n(1+\pi) \Phi_{2}^{2} \delta^{2}}{\eta_{2} n \pi \Phi_{2}^{2} \delta^{2}}\right\rceil
\end{aligned}
$$

and $k_{j}=k_{0}+j \theta, \forall j \in \mathbb{N}$, then we get

$$
\begin{array}{r}
x(k) \in \widetilde{R}_{j} \\
=\left\{x(k): E\left\{x^{\top}(k) P x(k)\right\} \leq \lambda_{\min }(P)\left(\frac{w_{j}}{1-\delta}\right)^{2}\right\}, \\
\forall k \in\left[k_{j}, k_{j+1}\right),
\end{array}
$$

and thus $\lim _{k \rightarrow \infty} E\left\{\|x(k)\|^{2}\right\}=0$. The proof is completed.

\section{Analysis and Comparison}

The aim of this section is analyzing and comparing the above two theorems, which includes the following four items.

(1) It can be seen from the proof process of above two theorems that the two event-driven schemes (5a) and (5b) proposed here can effectively simplify the system stability analysis, which means that (5a) and (5b) are with specific high practicability. In addition, from the theorem derivation process, we see that the analysis process under the eventdriven scheme (5b) is simpler than the one under (5a). 
(2) Both the parameters given in Theorems 2 and 3 can ensure the system stability. But, by comparing conditions (10) and (35), we know that the result of Theorem 2 must be more conservative than the one of Theorem 3 from the point of view of the maximum allowable packet loss rate.

(3) Contrasting (11a), (11b), (36a), and (36b) gives the fact that, in general, $\rho(\theta)$ defined in Theorem 3 is less (greater) than the one in Theorem 2. This means that the decreasing frequency of the mean squared value of the system state in Theorem 3 is slower than that of Theorem 2, but the decreasing rate in Theorem 3 is higher than the one in Theorem 2. Therefore, since the convergence time is affected by the combined effect of the decreasing frequency and the decreasing rate, we can not directly compare the magnitude of the convergence time between Theorems 2 and 3 . The convergence time will be compared by simulation in Section 6.

(4) Obviously, for any given $m \in \mathbb{N}$, if $\gamma(k)$ defined in event-driven schemes (5a) and (5b) is changed as

$$
\begin{aligned}
\gamma(k)=m \sqrt{n} \frac{\delta}{1-\delta} w_{l}, \\
\quad \forall k \in\left[k_{0}+l \theta, k_{0}+(l+1) \theta\right), l \in \mathbb{N} \cup\{0\},
\end{aligned}
$$

then the stability analysis process can still be simplified. Taking Theorem 3 as an example, if $\gamma(k)$ is defined by (44), then the variables $\rho$ and $\theta$ defined by (36a) and (36b) should be changed as

$$
\begin{aligned}
& \rho=\sqrt{\frac{\lambda_{\max }(P)}{\lambda_{\min }(P)}} \sqrt{m n(1+\pi)} \Phi_{2} \delta<1, \\
& \theta=\lceil\widetilde{\theta}\rceil=\left\lceil\frac{\lambda_{\min }(P)-\lambda_{\max }(P) m n(1+\pi) \Phi_{2}^{2} \delta^{2}}{\eta_{2} m n \pi \Phi_{2}^{2} \delta^{2}}\right\rceil
\end{aligned}
$$

which means that if $m>1$, comparing with the case of $m=1$, we have

(i) the range of values of $\delta$ becoming smaller;

(ii) the decreasing frequency of the mean squared value of the state becoming faster; that is, $\theta$ decreases;

(iii) The decreasing rate of the mean squared value of the state decreasing; that is, $\rho$ increases.

Summarized above, comparing with Theorem 3, if $\gamma(k)$ is defined by (44) with $m>1$, then item (i) above tells us that the range of values of quantizer parameters is more conservative. Moreover, items (ii) and (iii) show that the increasing of $m$ is a double-edged sword, and thus the value of $m$ and the actual convergence time can not be established a direct proportional relationship. To illustrate the effect of the variable $m$ on the system stability, we will compare the state mean square curve and analyze the convergence speed under different values of $m$ in the simulation.

\section{Simulation}

A well-known benchmark example shown below is adopted here to represent the usefulness of the main results:

$$
\dot{x}(t)=A_{c} x(t)+B_{c} u(t)
$$

with

$$
\begin{aligned}
& A_{c}:=\left[\begin{array}{cccc}
1.380 & -0.208 & 6.715 & -5.676 \\
-0.581 & -4.290 & 0 & 0.675 \\
1.067 & 4.273 & -6.654 & 5.893 \\
0.048 & 4.273 & 1.343 & -2.104
\end{array}\right], \\
& B_{c}:=\left[\begin{array}{cc}
0 & 0 \\
5.679 & 0 \\
1.136 & -3.146 \\
1.136 & 0
\end{array}\right] .
\end{aligned}
$$

By selecting the discretization interval as 0.1 , the continuoustime model above can be converted to the discrete-time one given as

$$
x(k+1)=A x(k)+B u(k)
$$

with

$$
\begin{aligned}
& A:=\left[\begin{array}{cccc}
1.1782 & 0.0015 & 0.5116 & -0.4033 \\
-0.0515 & 0.6619 & -0.0110 & 0.0613 \\
0.0762 & 0.3351 & 0.5606 & 0.3824 \\
-0.0006 & 0.3353 & 0.0893 & 0.8494
\end{array}\right], \\
& B:=\left[\begin{array}{ll}
0.0045 & -0.0876 \\
0.4672 & 0.0012 \\
0.2132 & -0.2353 \\
0.2131 & -0.0161
\end{array}\right] .
\end{aligned}
$$

If the initial state is selected as $x_{0}=\left[\begin{array}{llll}2 & 0 & 1.5 & 1\end{array}\right]^{\top}$, and other variables are selected as $\widetilde{w}(0)=6, N=2^{M}=2^{12}$, and $\varphi$ given in Table 1 , we can obtain the variables including $\theta, \rho, k_{0}$, and the applicable scope of $M$ and $\beta$ under Theorems 2 and 3, respectively. The total transmission times between the 9000 iterations can also be obtained. Moreover, the mean square values of the states can be shown in Figure 1, from which the mean square stability is guaranteed, and the convergence time can also be estimated.

Comparison of Theorems 2 and 3. From Table 1, we see that

(i) the applicable scope of Theorem 3 is larger than the one of Theorem 2 due to the fact that it can tolerate the smaller coding length $M$ and the larger packet loss rate $\beta$;

(ii) the convergence time under Theorem 3 is less than the one under Theorem 2, which means that Theorem 3 is better than Theorem 2 from the system performance point of view. However, Theorem 3 needs more transmission times than Theorem 2 to ensure the stability, which represents the fact 


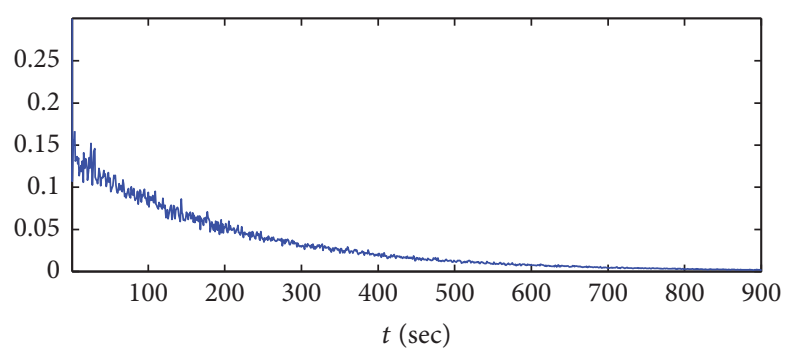

- $E\left\{\|x(t)\|^{2}\right\}$ under Theorem 2

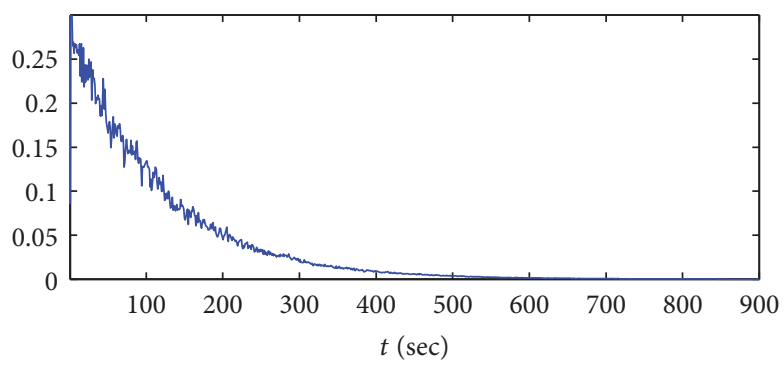

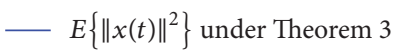

FIGURE 1: Comparison of mean square stability under different theorems.

TABLE 1: Comparison of the parameters in Theorems 2 and 3.

\begin{tabular}{lcccccccc}
\hline & $\theta$ & $\rho$ & $\begin{array}{c}\text { Convergence } \\
\text { time }\end{array}$ & $\begin{array}{c}\text { Transmission } \\
\text { times }\end{array}$ & $k_{0}$ & $\varphi$ & $\begin{array}{c}\text { Applicable } \\
\text { scope of } M\end{array}$ & $\begin{array}{c}\text { Applicable } \\
\text { scope of } \beta\end{array}$ \\
\hline Theorem 2 & 15 & 0.9928 & 800 & 2167 & 6 & 50 & $\geq 10$ & $\leq 0.40$ \\
Theorem 3 & 26 & 0.9777 & 540 & 2548 & 3 & 20 & $\leq 8$ & $\leq 0.44$ \\
\hline
\end{tabular}

TABLE 2: Comparison of the parameters under different $m$.

\begin{tabular}{ccccccccc}
\hline & $\theta$ & $\rho$ & $\begin{array}{c}\text { Convergence } \\
\text { time }\end{array}$ & $\begin{array}{c}\text { Transmission } \\
\text { times }\end{array}$ & $k_{0}$ & $\varphi$ & $\begin{array}{c}\text { Applicable } \\
\text { scope of } M\end{array}$ & $\begin{array}{c}\text { Applicable } \\
\text { scope of } \beta\end{array}$ \\
\hline$m=1$ & 26 & 0.9777 & 400 & 2548 & 3 & 20 & $\leq 8$ & $\leq 0.44$ \\
$m=4$ & 7 & 0.9943 & 500 & 2276 & 5 & 60 & $\leq 10$ & $\leq 0.44$ \\
$m=8$ & 4 & 0.9971 & 600 & 1906 & 5 & 120 & $\geq 12$ & $\leq 0.44$ \\
\hline
\end{tabular}

that Theorem 2 is superior to Theorem 3 in terms of saving the network bandwidth.

Comparison of System Performance under Different Values of $m$. To illustrate the effect of different values of $m$ defined in (44) on system performance, we select $m=1,4,8$ and show the relating variables and state trajectories in Table 2 and Figure 2, respectively.

From Table 2, we know the following:

(i) With the increase of $m$, the applicable scope of the theorem is reduced; that is, the allowable lower bound of $M$ is increased.

(ii) When $m$ increases, the decreasing interval $\theta$ decreases but the decreasing rate $\rho$ increases, under the coupling actions of which the convergence time is prolonged. However, the transmission times are reduced as long as $m$ increases.
Therefore, in the premise of ensuring the convergence of the system, if it takes the minimum transmission times as the goal, then the value of $m$ should be increased. Otherwise, if the goal is increasing convergence rate or the applicable scope of the algorithm, then $m$ should be reduced appropriately.

\section{Conclusion}

The mean square stability of NCSs with data quantization, packet losses, and event-driven communication is studied in this paper. Some sufficient conditions are given to ensure the mean square stability of the closed-loop system. From Figures 1 and 2 , it is seen that the convergence speed of the state trajectory is low due to the large value of $\rho$; how to improve it is one of our future research directions. 

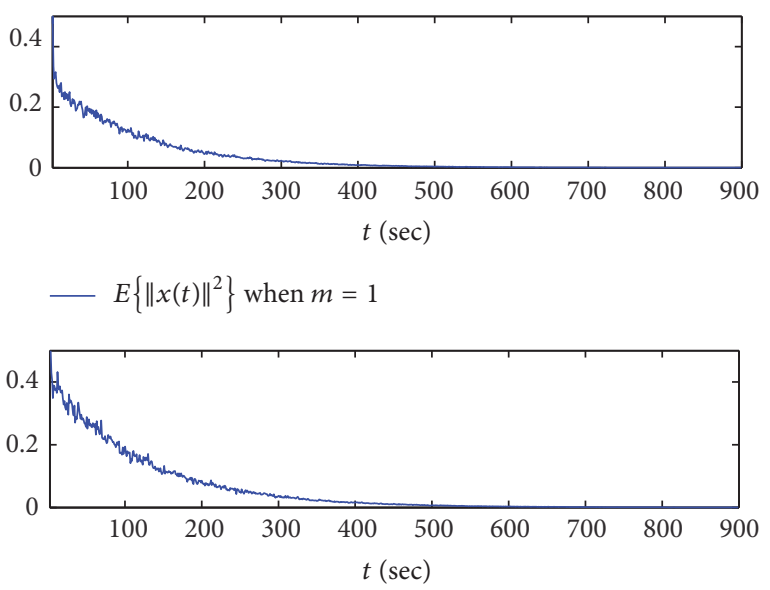

— $E\left\{\|x(t)\|^{2}\right\}$ when $m=4$

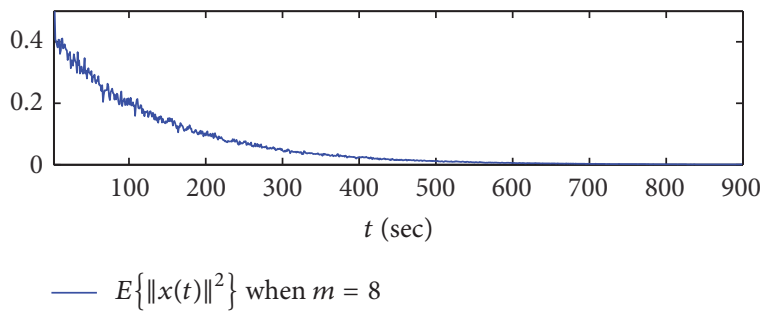

FIGURE 2: Comparison of mean square stability under different values of $m$.

\section{Conflicts of Interest}

The author declares that they have no conflicts of interest.

\section{Acknowledgments}

The work was supported by the National Natural Science Foundation of China (61403125, 61773154, 41501562, 61304258, 61503123, and U1504616), the Natural Science Foundation of Henan Province Education Department (15A413012), and the Fundamental Research Funds for the Henan Provincial Colleges and Universities in Henan University of Technology (2015RCJH15).

\section{References}

[1] M. Ji, Y. Cai, and W. Zhang, "Stabilization of discrete-time linear systems with quantization and arbitrary packet losses," Transactions of the Institute of Measurement and Control, vol. 37, no. 9, pp. 1084-1094, 2015.

[2] M. Ji, Z. Li, and W. Zhang, "Quantized feedback stabilization of discrete-time linear system with markovian jump packet losses," Neurocomputing, vol. 158, pp. 307-314, 2015.

[3] F.-L. Qu, B. Hu, Z.-H. Guan, Y.-H. Wu, D.-X. He, and D.-F. Zheng, "Quantized stabilization of wireless networked control systems with packet losses," ISA Transactions, vol. 64, pp. 92-97, 2016.

[4] Q. Ling, "A sufficient bit rate condition for mean-square stabilisation of linear systems over multiple lossy networks," IET Control Theory \& Applications, vol. 10, no. 13, pp. 1531-1538, 2016.
[5] X.-W. Jiang, X.-H. Zhang, Z.-H. Guan, L. Yu, and H.-C. Yan, "Performance limitations of networked control systems with quantization and packet dropouts," ISA Transactions, vol. 67, pp. 98-106, 2017.

[6] Q.-L. Han, Y. Liu, and F. Yang, "Optimal communication network-based $H_{\infty}$ quantized control with packet dropouts for a class of discrete-time neural networks with distributed time delay," IEEE Transactions on Neural Networks and Learning Systems, vol. 27, no. 2, pp. 426-434, 2016.

[7] M. S. Mahmoud and M. H. Baig, "Networked feedback control for systems with quantization and non-stationary random delays," IMA Journal of Mathematical Control and Information, vol. 32, no. 1, pp. 119-140, 2015.

[8] M. S. Mahmoud and N. B. Almutairi, "Feedback fuzzy control for quantized networked systems with random delays," Applied Mathematics and Computation, vol. 290, pp. 80-97, 2016.

[9] S. Jiang and H. Fang, "Quantized stabilization of discrete-time systems in a networked environment," Applied Mathematical Modelling, vol. 38, no. 5-6, pp. 1685-1697, 2014.

[10] W.-C. Hsu, L.-W. Lee, K.-H. Tseng, C.-Y. Lu, C.-W. Liao, and H.-N. Shou, "Design of feedback control for networked finite-distributed delays systems with quantization and packet dropout compensation," Discrete Dynamics in Nature and Society, Article ID 158972, Art. ID 158972, 15 pages, 2015.

[11] M. Li, J. Sun, and L. Dou, "Stability of an improved dynamic quantised system with time-varying delay and packet losses," IET Control Theory and Applications, vol. 9, no. 6, pp. 988-995, 2015.

[12] R. Lu, P. Yang, J. Bai, and A. Xue, "Quantized observer-based sliding mode control for networked control systems via the time-delay approach," Circuits, Systems, and Signal Processing, vol. 35, no. 5, pp. 1563-1577, 2016.

[13] S. Wang, M. Yu, H. Wang, and W. Tan, "Switched quantization level control of networked control systems with packet dropouts," Mathematical Problems in Engineering, vol. 2014, Article ID 890543, pp. 1-8, 2014.

[14] J. Yu, L. Nan, X. Tang, and P. Wang, "Model predictive control of non-linear systems over networks with data quantization and packet loss," ISA Transactions, vol. 59, pp. 1-9, 2015.

[15] Y. Zou, J. Lam, Y. Niu, and D. Li, "Constrained predictive control synthesis for quantized systems with Markovian data loss," Automatica, vol. 55, pp. 217-225, 2015.

[16] M. Li, L. Dou, J. Sun, and D. Wang, "Dynamic quantized predictive control for systems with time-varying delay and packet loss in the forward channel," Mathematical Problems in Engineering, vol. 2015, Article ID 596916, pp. 1-11, 2015.

[17] J. Yan, Y. Xia, B. Liu, and M. Fu, "Stabilisation of quantised linear systems with packet dropout," IET Control Theory \& Applications, vol. 5, no. 8, pp. 982-989, 2011.

[18] J. Yan, Y. Xia, and L. Li, "Stabilization of fuzzy systems with quantization and packet dropout," International Journal of Robust and Nonlinear Control, vol. 24, no. 10, pp. 1563-1583, 2014.

[19] J. Yan and Y. Xia, "Quantized control for networked control systems with packet dropout and unknown disturbances," Information Sciences, vol. 354, pp. 86-100, 2016.

[20] E. Konaka, "Stability of networked control system with dynamic quantizer and event-driven zero-order hold," in Proceedings of the SICE (Society of Instrument and Control Engineers) Annual Conference (SICE '07), pp. 1892-1897, Takamatsu, Japan, September 2007. 
[21] H. Yu and P. J. Antsaklis, "Quantized output synchronization of networked passive systems with event-driven communication," in Proceedings of the 2012 American Control Conference (ACC), pp. 5706-5711.

[22] D. Du, B. Qi, M. Fei, and Z. Wang, "Quantized control of distributed event-triggered networked control systems with hybrid wired-wireless networks communication constraints," Information Sciences, vol. 380, pp. 74-91, 2017.

[23] F.-L. Qu, Z.-H. Guan, D.-X. He, and M. Chi, "Event-triggered control for networked control systems with quantization and packet losses," Journal of the Franklin Institute, vol. 352, no. 3, pp. 974-986, 2015.

[24] H. Yang, Y. Xu, and J. Zhang, "Event-driven control for networked control systems with quantization and Markov packet losses," IEEE Transactions on Cybernetics, vol. 47, no. 8, pp. 2235-2243, 2017.

[25] N. Elia and S. K. Mitter, "Stabilization of linear systems with limited information," IEEE Transactions on Automatic Control, vol. 46, no. 9, pp. 1384-1400, 2001.

[26] M. Fu and L. Xie, "The sector bound approach to quantized feedback control," IEEE Transactions on Automatic Control, vol. 50, no. 11, pp. 1698-1711, 2005.

[27] D. Liberzon, "Hybrid feedback stabilization of systems with quantized signals," Automatica, vol. 39, no. 9, pp. 1543-1554, 2003.

[28] J. Yan, "Parameters design for logarithmic quantizer based on zoom strategy," Journal of Control Science and Engineering, vol. 2017, Article ID 4028258, pp. 1-9, 2017. 


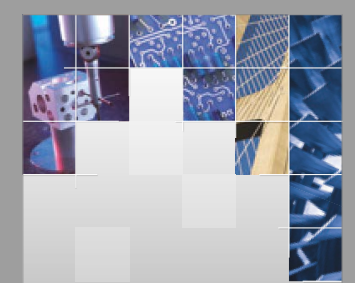

\section{Enfincering}
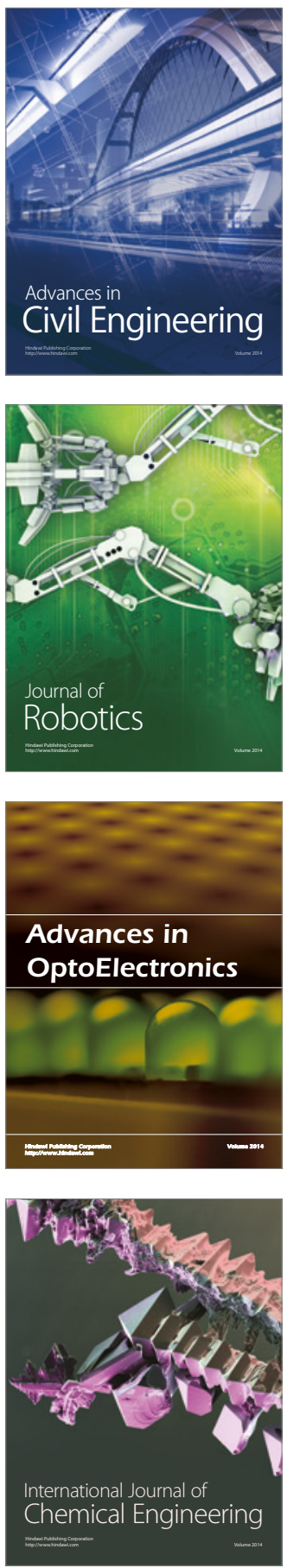

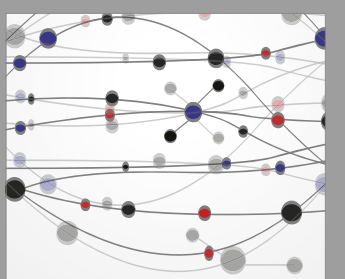

The Scientific World Journal

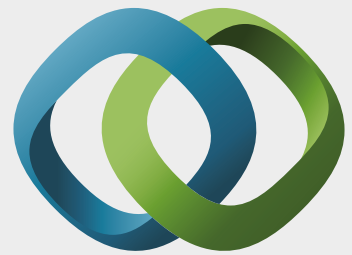

\section{Hindawi}

Submit your manuscripts at

https://www.hindawi.com
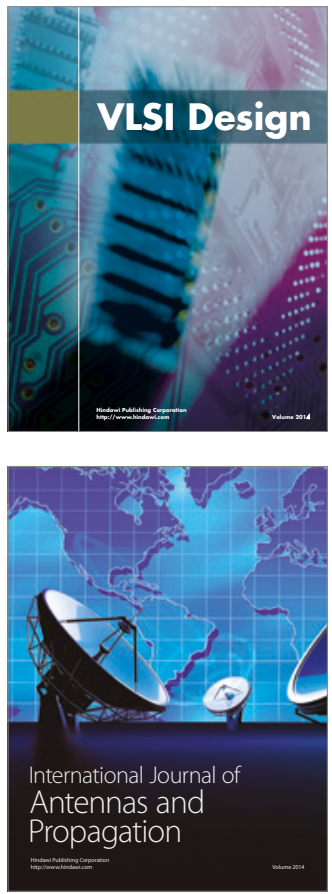

\section{Rotating}

Machinery
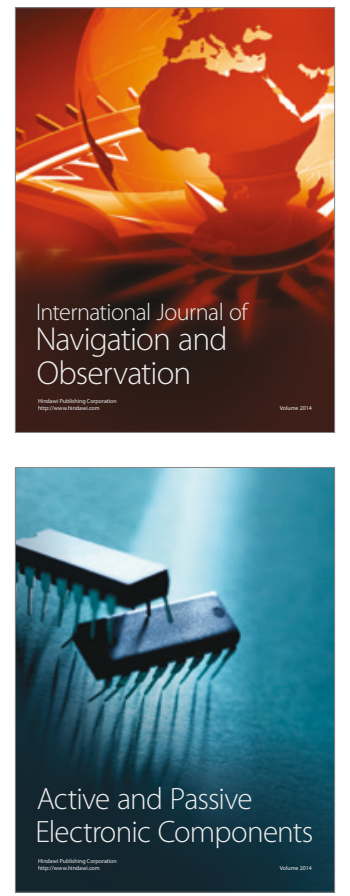
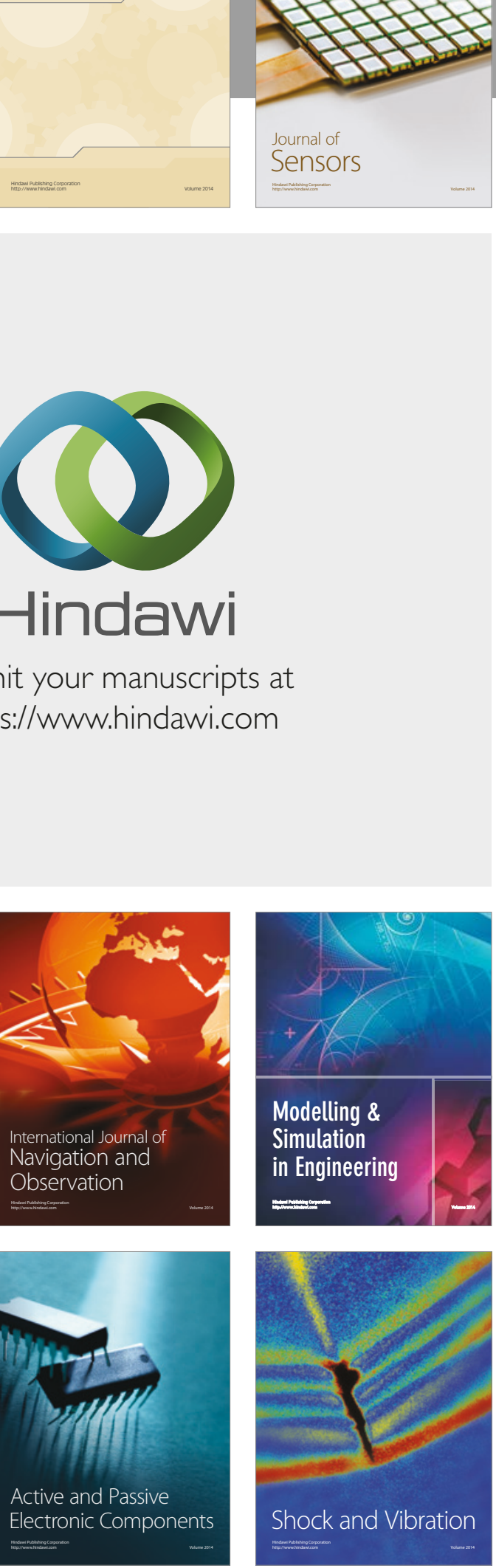
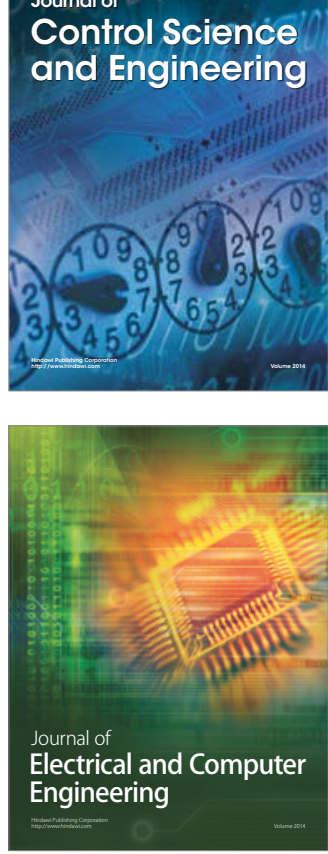

Distributed

Journal of

Control Science

and Engineering
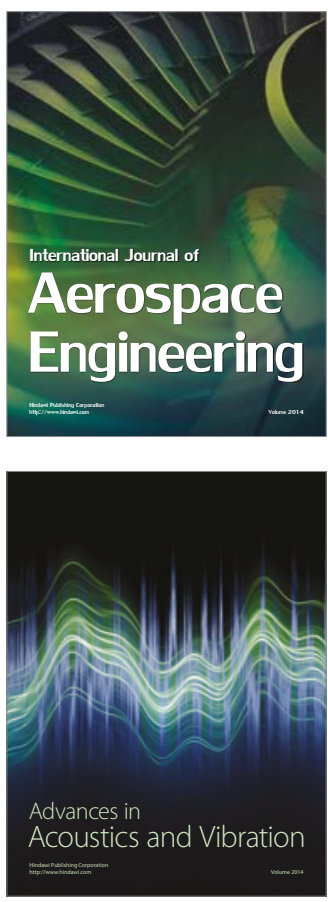

Sensor Networks 